\title{
Theory of optically-triggered electrical breakdown of semiconductors
}

\author{
Kenneth E. Kambour ${ }^{1}$, Harold P. Hjalmarson ${ }^{2}$ and Charles W. Myles ${ }^{1}$ \\ ${ }^{1}$ Department of Physics, Texas Tech University, Lubbock, TX 79409-1051 USA \\ ${ }^{2}$ Sandia National Laboratories, Albuquerque, NM 87185-0316
}

\begin{abstract}
In this paper, we describe a rate equation approach that leads to new insights about electrical breakdown in insulating and semiconducting materials. In this approach, the competition between carrier generation by impact ionization and carrier recombination by Auger and defect recombination leads to steady state solutions for the carrier generation rate, and it is the accessibility of these steady state solutions, for a given electric field, that governs whether breakdown does or does not occur. This approach leads to theoretical definitions for not only the intrinsic breakdown field but also other characteristic quantities. Results obtained for GaAs using a carrier distribution function calculated by both a Maxwellian approximation and an ensemble Monte Carlo method will be discussed.
\end{abstract}

\section{Introduction}

Electrical breakdown of insulators occurs if the applied electric field exceeds a threshold field, the breakdown field. At fields near and above this field, the electrical current grows dramatically. This growing electrical current heats the insulator and leads to its destruction if unchecked.

Much effort has been focused on understanding breakdown of solid insulators [1, 2]. An important theoretical foundation was the avalanche breakdown theory formulated for electrical breakdown of insulating gases [3]. In this theory, applied to solids, an initial electron is heated by the field until it has achieved enough energy to create another electron by impact ionization. The Joule heating of this initial electron by the field competes with cooling by phonon emission. This process leads to an "avalanche" of electron-hole pairs, assuming that the electrode separation is large enough to allow a carrier to gain enough energy from the electric field to reach the energy for impact ionization.

Extensive experimental work has focused on determining the ultimate breakdown field for an insulator $[1,2]$. This idealized characteristic has been elusive because electrical breakdown at a lower field may be triggered by imperfections in the sample. Furthermore, breakdown is a probabilistic process even for an ideal sample because it is triggered by random fluctuations in carrier density.
Another approach to the study of breakdown is to induce it by injecting carriers. In this way, the breakdown conditions can be controlled. This approach has been undertaken during the development of optically-triggered photoconductive semiconductor switches (PCSS's) [4-6]. Such switches are fabricated by attaching electrical contacts to a bulk semiconductor, usually GaAs or Si [4-6].

In studies of electrical breakdown in these devices, the breakdown can be triggered into a steady state condition in which the carrier density, carrier temperature, and the electric field remain approximately constant [4-6]. In this condition, the current flows in filaments, and it is sustained by a field, called the "lockon" field, that is much lower than the intrinsic breakdown field (the breakdown duration can be limited to avoid heating of the lattice) [4-7].

In this paper, we develop a theory that explains the properties of such steady state electrical breakdown. We start with a kinetic equation governing the growth of carrier density. In this equation, the dominant contribution is impact ionization. We find that breakdown occurs if the impact ionization rate increases with increasing carrier density, and our qualitative solutions suggest that such behavior is necessary in a viable electrical breakdown theory. Furthermore, by solving the Boltzmann equation using an ensemble Monte Carlo (EMC) technique, we show that inclusion of carrier-carrier scattering produces solutions similar to the qualitative solutions. Analysis of these resultant steady state solutions allows us to define the breakdown field and the lock-on field and to understand the conditions that lead to breakdown.

\section{Theory}

Our approach is to write an expression for the rate of change of carrier density in terms of the difference between the carrier generation and recombination processes. The key carrier generation process is impact ionization as in all other theories of electrical breakdown [1-3]. The reverse process, Auger recombination, is also included [8]. Finally, as will be discussed, recombination at defect centers must also be included. 
The rate of change of carrier density $n$ for these three processes can be written as an integral over the first Brillioun zone as

$$
\frac{d n}{d t}=\int f_{k}\left[r_{i i}-r_{\text {Auger }}-r_{\text {defects }}\right] d^{3} k
$$

in which $f_{k}$ is the carrier distribution function and the rates are impact ionization, Auger recombination and defect recombination, respectively.

\section{A. Qualitative Solution}

Since we know the forms for each term in (1), we can do a qualitative analysis to gain physical insight before we do the EMC calculations. Thus we write [1$3,8]$

$$
\frac{d n}{d t}=R(n, F) n
$$

with

$$
R(n, F)=C(F, n)-a n^{2}-r .
$$

In this expression, $F$ is the electric field and $C(F, n), a$, and $r$ are the impact ionization, Auger, and defect recombination rate coefficients. We are interested in non-trivial steady state solutions to (2), and thus we consider the case

$$
R(n, F)=C(F, n)-a n^{2}-r=0
$$

In agreement with previous work, we first assume that the impact ionization is independent of carrier density. Then $C(F, n)=\mathrm{C}(F)$, and we solve (3) for the steady-state carrier density $n_{s s}(F)$ :

$$
n_{s s}(F)=\sqrt{\frac{C(F)-r}{a}} .
$$

Using the common approximation that $\mathrm{C}(F)=\alpha e^{-\beta / \mathrm{F}}(\alpha$ and $\beta$ are parameters), we plot the resultant $n(F)$ as the dashed curve in Figure 1. In that figure, the intrinsic breakdown field, $\mathrm{F}_{\mathrm{B}}$, is defined as the field for which $n\left(F_{B}\right)=0$. Using the original rate equation, we observe that $R(F, n) \leq 0$ if $F \leq F_{B}$ and $R(F, n)>0$ if $F>F_{B}$. Thus an infinitesimal carrier density will grow only if the applied field exceeds the breakdown field, $F_{B}$. Furthermore, we observe that defect recombination must be considered; otherwise breakdown could occur at an arbitrarily small field if the sample size is large enough.

The solution becomes qualitatively different if we allow the impact ionization rate to increase with increasing carrier density. Such behavior is expected at high carrier density because carrier-carrier scattering makes the distribution function more Maxwellian [10, 11]. As a result, a larger fraction of the carriers are in the high energy tail of the distribution function that produces impact ionization. For this to occur, the carrier density must be high enough that carrier-carrier scattering dominates phonon scattering $[9,2]$. For this qualitative analysis we assume a linear dependence, $C(F, n)=C(F)\left(1+n / n_{0}\right)$, in which $n_{0}$ is a constant. For this case, the condition $\mathrm{R}(\mathrm{n}, \mathrm{F})=0$ produces a quadratic equation, and its solution is shown schematically as the solid curve in Figure 1.

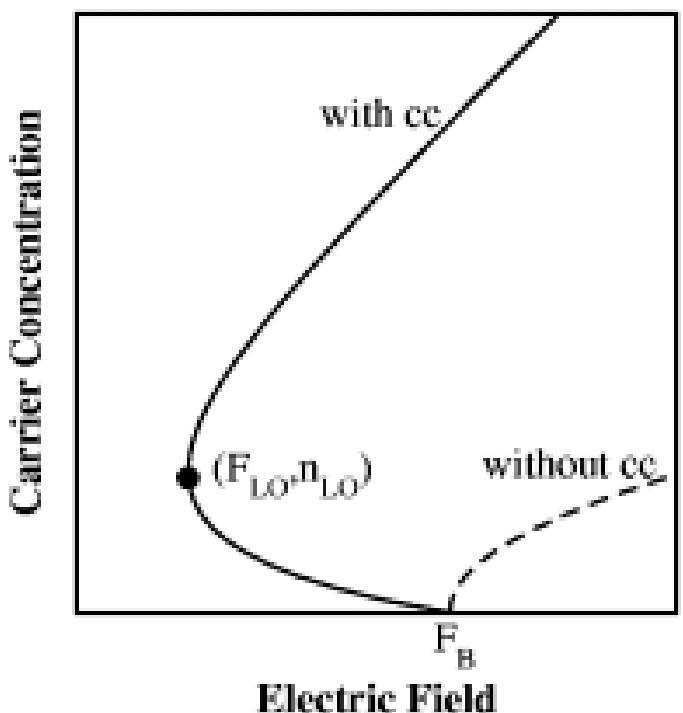

Figure 1: Schematic showing breakdown with and without carrier-carrier scattering.

As can be seen in Figure 1, including the effects of carrier-carrier scattering changes the solution in a qualitative way. It introduces a new minimum field that sustains the breakdown, and we define this field as the lock-on field, $\mathrm{F}_{\mathrm{LO}}$ (see Figure 1). The original breakdown field $F_{B}$ and the breakdown conditions remain unchanged. However, in this case we observe that the carrier density will increase by a finite value if breakdown occurs. We note that the new steady state carrier density following breakdown depends on the external electric circuit. Furthermore, we observe that if carrier-carrier scattering is not included, then the breakdown condition does not lead to a large growth in carrier density. Thus we assert that carrier-carrier scattering or an equivalent phenomenon must be included in a theory of electrical breakdown.

Figure 1 also shows that carrier injection can stimulate electrical breakdown. This figure shows that $\mathrm{R}>0$ if the injected carrier density is sufficiently large and if $\mathrm{F}>\mathrm{F}_{\mathrm{LO}}$. However, if $\mathrm{F}<\mathrm{F}_{\mathrm{LO}}$ the injected charge, no matter how large, will always decay to zero because the electric field is not large enough to sustain breakdown. 


\section{B. Quantitative Calculations}

In addition to the computationally intensive EMC calculations to implement carrier-carrier scattering, we performed two other types of calculations designed to represent the low and high carrier density extremes. For low carrier densities, the effects of carrier-carrier scattering are minimal and a standard Monte Carlo (MC) simulation without carrier-carrier scattering is used to evaluate the right hand side of (1).

The second type of calculations is based on the fact that a quasi-equilibrium steady state will be achieved at high densities. In this state the carrier generation and recombination rates must be approximately equal and the Joule heating and phonon cooling rates must also be approximately equal. Thus, in a high-density implementation, the distribution becomes a quasiequilibrium Maxwellian distribution characterized by a carrier temperature $\mathrm{T}_{\mathrm{c}}$.

For this situation, the steady state energy balance condition is

$$
q v_{d} F=B\left(T_{c}\right)
$$

in which $v_{d}$ is the drift velocity, $q$ is the charge, and $B\left(\mathrm{~T}_{\mathrm{c}}\right)$ is the phonon-carrier cooling rate. The calculation of $B\left(\mathrm{~T}_{\mathrm{c}}\right)$ for a Maxwellian distribution is discussed in [12]. Using the requirement that the net carrier generation rate is zero, it is possible to find $\mathrm{T}_{\mathrm{c}}$ for a given value of $n$. Then, using Eq. (6), $\mathrm{T}_{\mathrm{c}}$ can be used to calculate $F$.

\section{Results}

\section{A. Model Material}

In order to test the theory, we first applied it to a model material with single parabolic conduction and valence bands and a band gap of $1.5 \mathrm{eV}$. Both bands had effective mass $0.5 m_{e}$. To simplify the calculation for this model material we also allowed only acoustic deformation potential scattering.

Figure 2 shows numerical results for the steady state carrier density of the model material as a function of field. These solutions are very similar to the schematic solutions shown in Figure 1. By inspection, Figure 2 shows a lock-on field, $F_{\mathrm{LO}} \cong 30 \mathrm{kV} / \mathrm{cm}$, the lowest field for which there is a non-trivial steady state solution. If $F$ $<\mathrm{F}_{\mathrm{LO}}$, any injected charge will decay to zero. If $F>$ $\mathrm{F}_{\mathrm{LO}}$, then the injected charge can grow. For example if $F=35 \mathrm{kV} / \mathrm{cm}$, then it either decays until $n=0$ or grows until $n=1.8 \times 10^{19} \mathrm{~cm}^{-3}$.

Figure 2 also shows the intrinsic breakdown field, the field where the Monte Carlo results without carriercarrier scattering first have a non-zero solution, approximately $40 \mathrm{kV} / \mathrm{cm}$. Once this field is reached, an arbitrarily small carrier density will produce more carriers until the system reaches a steady state, and the carrier density reaches a maximum value given by the upper curve. Earlier breakdown theories implicitly assume that at the breakdown field the carrier density will increase to infinity $[1,2]$. By contrast, our theory predicts that the carrier density at breakdown will be large but limited by Auger recombination.

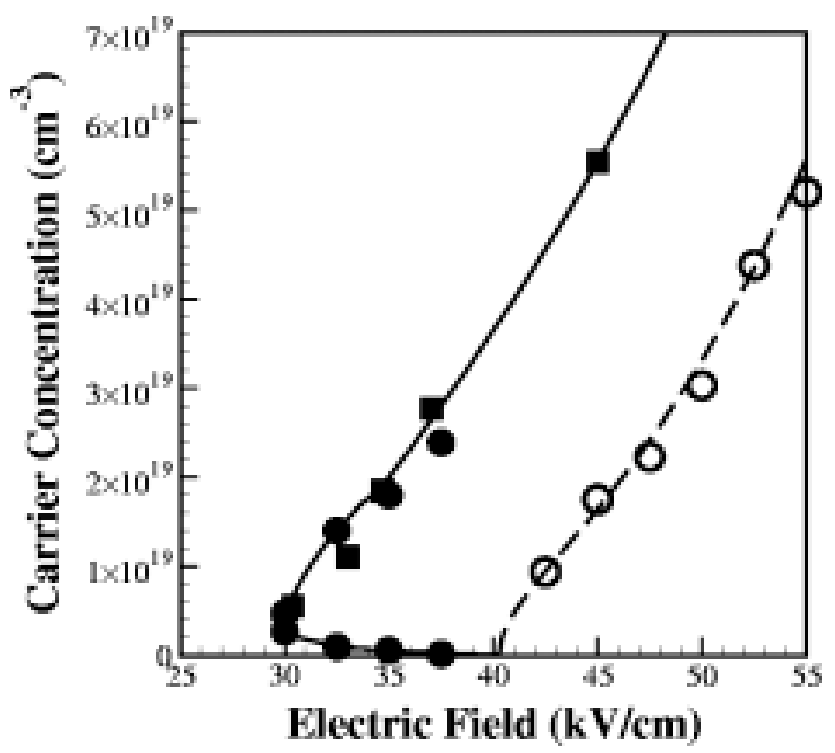

Figure 2: Breakdown plot for the model material. The hollow circles are MC results without carruercarrier scattering, the solid circles are EMC results with carrier-carrier scattering, and the squares are Maxwellian results. The solid and dashed curves are meant to guide the eye.

\section{B. GaAs, InP, and Si}

We have done similar calculations for GaAs, InP, and Si. For these materials we have used electronic bandstructures computed in the local pseudopotential approximation [13]. For Si, we used improved form factors [14].

The GaAs results are shown in Figure 3. This figure shows that the intrinsic breakdown field is $175 \mathrm{kV} / \mathrm{cm}$ and the lock-on field is $90 \mathrm{kV} / \mathrm{cm}$. The calculations for InP predict a breakdown field of $168 \mathrm{kV} / \mathrm{cm}$ and a lockon field of $40 \mathrm{kV} / \mathrm{cm}$. The experimental values for the lock-on fields are 5 to $10 \mathrm{kV} / \mathrm{cm}$ for GaAs [4-6] and $14.4 \mathrm{kV} / \mathrm{cm}$ for InP [15]. The calculations for Si predict a lock-on field of $77 \mathrm{kV} / \mathrm{cm}$ and a breakdown field of $104 \mathrm{kV} / \mathrm{cm}$; experiments have not observed lock-on in Si [4]. 




Figure 3: GaAs results.

\section{Summary and Conclusions}

We have developed a theory of electrical breakdown in which impact ionization is balanced by Auger and defect recombination. This theory leads to definitions of the breakdown and sustaining (lock-on) fields. It has led to numerical predictions for these fields in GaAs and $\mathrm{InP}$, and these fields have been compared with experimental values. This theory predicts that lock-on will occur in both GaAs and InP, in qualitative agreement with experiment. The predicted InP lock-on field is in reasonable quantitative agreement with experiment. We also predict that lock-on in $\mathrm{Si}$ will be experimentally difficult to observe.

\section{References}

[1] S. Whitehead, Dielectric Breakdown of Solids, London: Oxford University Press, 1951.

[2] J. J. O'Dwyer, The Theory of Dielectric Breakdown of Solids. London: Oxford University Press, 1964.

[3] H. Raether, Electron Avalanches and Breakdown of Gases. London: Buttersworths, 1964.

[4] A. Rosen and F. J. Zutavern, eds., High Power Optically Activated Solid State Switches, Artech House, Boston, 1994.

[5] G. M. Loubriel, M. W. O'Malley, and F. J. Zutavern, "Toward pulsed power uses for photoconductive semiconductor switches: closing switches", in Proc. 6th IEEE Pulsed Power Conf., 1987, p. 145.

[6] G. M. Loubriel, F. J. Zutavern, H. P. Hjalmarson, and M. O'Malley, "Closing photoconductive semiconductor switches", in Proc. 7th IEEE Pulsed Power Conf., 1989, p. 365.

[7] F. J. Zutavern and G. M. Loubriel, "High-voltage lateral switches from silicon or gallium arsenide" in High Power
Optically Activated Solid State Switches, Artech House, Boston, 1994, p. 245.

[8] A. R. Beattie and P. T. Landsberg, "Auger effect in semiconductors", Proc. Phys. Soc. A, 249, p. 16, 1959.

[9] R. Stratton, "The influence of interelectronic collisions on conduction and breakdown in polar crystals", Proc. Roy. Soc. A, 246, p. 406, 1958.

[10] H. P. Hjalmarson, F. J. Zutavern, G. M. Loubriel, A. Baca, K. Khachaturyan, and D. R. Wake, "An impact ionization model for optically-triggered current filaments in GaAs", SAND93-3972, 1991.

[11] H. P. Hjalmarson, G. M. Loubriel, F. J. Zutavern, D. R. Wake, S. Kang, K. Kambour,and C. W. Myles, "A collective impact ionization theory of lock-on", in Proc. 12th IEEE Pulsed Power Conf., 1999, p. 299.

[12] K. Kambour, S. Kang, C. W. Myles, and H.P. Hjalmarson, "Steady-state properties of lock-on current filaments in GaAs", IEEE Trans. Plasma Science, 28, p. 1497, 2000.

[13] M. Cohen and T. Bergstresser, "Band structures and pseudopotential form factors for fourteen semiconductors of the diamond and zinc-blende structures", Phys. Rev., 141, p. 789, 1969.

[14] T. Kunikiyo, M. Takenaka, Y. Kamakura, M. Yamaji, H. Mizuno, M. Morifuji, K. Taniguchi and C. Hamaguchi, "A Monte Carlo simulation of anisotropic electron transport in silicon including full band structure and anisotropic impactionization model", J. Appl. Phys., 75, p. 297, 1994.

[15] F.J. Zutavern, G. M. Loubriel, M. W. O'Malley, L. P. Shanwald, W. D. Helgeson, D. L. McLaughlin, and B. B. McKenzie, "Photoconductive semiconductor switch experiments for pulsed power applications", IEEE Trans. Elect. Dev., 37, p. 2472, 1990.

Author address: Harold P. Hjalmarson, Sandia National Laboratories, MS-0316, Albuquerque, NM 87185, USA, Email: hphjalm@sandia.gov. 